\title{
Increased Expression of Versican in the Inflammatory Response to UVB- and Reactive Oxygen Species-Induced Skin Tumorigenesis
}

\author{
Makoto Kunisada, ${ }^{*}$ Flandiana Yogianti, ${ }^{*}$ \\ Kunihiko Sakumi, ${ }^{\dagger}$ Ryusuke Ono, ${ }^{*}$ \\ Yusaku Nakabeppu, ${ }^{\dagger}$ and Chikako Nishigori* \\ From the Division of Dermatology, Department of Internal \\ Related, Kobe University Graduate School of Medicine, Kobe \\ University, Kobe; and the Division of Neurofunctional Genomics, ${ }^{\dagger}$ \\ Medical Institute of Bioregulation, Kyusyu University, Fukuoka, \\ Japan
}

Excessive exposure to UV radiation is a major risk factor for developing skin cancer. UV-induced reactive oxygen species (ROS) cause accumulation of DNA damage products such as 8-oxoguanine (8oxoG) in the skin. We have previously shown that mice lacking the repair enzyme 8-oxoguanine glycosylase (Ogg1 knockout mice) are highly susceptible to skin cancer after long-term UVB exposure. To investigate the genes involved, we performed gene profiling of Ogg1 knockout mouse skin after UVB exposure. Among the up-regulated genes in UVBtreated Ogg1 knockout mice, inflammatory response pathway-related genes were most affected. The Vcan gene, which encodes the large extracellular matrix proteoglycan versican, was continuously up-regulated in UVB-treated Ogg1 knockout mice, suggesting that versican is a mediator of skin cancer development. We examined the expression pattern of versican in skin tumors from wild-type mice and UVB-treated Ogg1 knockout mice, and also analyzed 157 sun-related human skin tumors. Versican was strongly expressed in malignant skin tumors in both mice and humans, and especially in Ogg1 knockout mice. Additionally, infiltrating neutrophils strongly colocalized with versican in UVBtreated Ogg1 knockout mouse skin. These data demonstrate that inflammatory responses, particularly neutrophil infiltration and versican up-regulation, are closely involved in UVB/ROS-induced skin tumorigenesis. (Am J Pathol 2011, 179:3056-3065; DOI: 10.1016/j.ajpath.2011.08.042)
The mechanism of sunlight-induced skin carcinogenesis has been extensively investigated, and direct DNA damage with subsequent generation of dipyrimidine photoproducts has been considered a major cause of skin cancer. ${ }^{1,2}$ On the other hand, UV irradiation is known to induce the generation of reactive oxygen species (ROS), which in turn cause various types of DNA damage. Among many oxidative DNA base modifications, 8-oxoguanine (8-oxoG) pairs with adenine as well as cytosine during DNA replication, resulting in $\mathrm{GC} \rightarrow \mathrm{TA}$ mutations. ${ }^{3-5}$ Accumulation of 8-oxoG has been shown in skin cells after irradiation with UVB (wavelength, 280 to 320 $\mathrm{nm}) .{ }^{6}$ UV-induced 8-oxoG has been recognized as responsible for development of skin cancers in both humans and animals. ${ }^{7,8}$

In mammalian cells, Ogg1 encodes a DNA glycosylase/apurinic/apyrimidinic (AP) lyase that is responsible for the excision of 8-oxoG from DNA. ${ }^{9}$ We showed that Ogg1 knockout mice exhibit impaired ability to excise 8-oxoG from DNA in epidermal cells after UVB exposure and that Ogg1 knockout mice develop significantly more skin tumors after long-term UVB irradiation than do wildtype or heterozygous mice. ${ }^{7}$ In addition, we found that the ratio and incidence of malignant skin tumors produced by chronic UVB irradiation in Ogg1 knockout mice is significantly higher and occurs at an earlier age than in wild-type or heterozygous mice. Nonetheless, the mechanisms underlying the highly susceptible phenotype of

Supported entirely by the Global Center of Excellence (GCOE) Program for "Education and Research on Signal Transduction Medicine in the Coming Generation" of Japan's Ministry of Education, Culture, Sports, Science and Technology (M.K. and C.N.) and by Health and Labor Science Research Grants for the Specified Disease Treatment Research Program (C.N.)

Accepted for publication August 22, 2011.

Supplemental material for this article can be found at http://ajp. amjpathol.org or at doi: 10.1016/j.ajpath.2011.08.042.

Address reprint requests to Chikako Nishigori, M.D., Ph.D., Division of Dermatology, Clinical Molecular Medicine, Faculty of Medicine Kobe University Graduate School of Medicine, Kobe 650-0017, Japan. E-mail: chikako@med.kobe-u.ac.jp. 
Ogg1 knockout mice for developing UVB-induced skin cancers remain to be fully elucidated.

In the present study, we performed gene profiling of UVB-irradiated skin to investigate the relevant genes involved in the susceptibility to skin cancer in Ogg1 knockout mice. We found that inflammatory pathway-related genes were most significantly affected among the upregulated genes in Ogg1 knockout mice. Among the continuously up-regulated genes in Ogg1 knockout mice after UVB exposure, the expression of Vcan (the gene encoding versican, a large chondroitin sulfate proteoglycan) significantly and persistently differed between wildtype and Ogg1 knockout mice after UVB exposure. Versican is a component of the extracellular matrix (ECM). It participates in the release of cytokines from the ECM and interacts with other ECM molecules, including collagen, proteoglycans, hyaluronan, and glycoproteins. ${ }^{10,11}$ We studied versican expression in skin tumors of wild-type and Ogg1 knockout mice after long-term UVB exposure, as well as in human sunlight-related skin tumors, and found that strong versican expression is highly associated with UV/ROS-related skin tumors. To study the role of versican in ROS-induced 8-oxoG accumulation, the inflammatory response, and the development of skin tumors, we further assessed the involvement of neutrophil infiltration in UVB-exposed wild-type and Ogg1 knockout mice.

\section{Materials and Methods}

\section{Mice}

C57BL/6J Ogg1 knockout mice ${ }^{12}$ and their respective wild-type counterparts were used for gene profiling of the skin and for immunohistochemical studies after UVB exposure. Mice aged 8 to 9 weeks were selected for all studies. We inbred Ogg1 heterozygous mice (C57BL/6J; $n=12$ ), and their genotype was determined as described previously. ${ }^{12}$ The mice were housed under special pathogen-free conditions, and all animal experiments were conducted according to the Guidelines for Animal Experimentation of the Kobe University School of Medicine.

\section{RNA Isolation, Gene Expression Profiling, and Real-Time Quantitative PCR}

RNAs from skin isolated at each time point were used for microarray and real-time PCR analyses. After genotyping, tissue samples were pooled into two separate groups per genotype for biological replicates. The duplicated RNAs were isolated according to a method described previously, ${ }^{13}$ and cyanine 5 -labeled cRNAs were generated and hybridized to the CodeLink Mouse Whole Genome Bioarray (35K; Filgen, Nagoya, Japan). Data were analyzed using the Microarray Data Analysis Tool software (version 3.2; Filgen). After screening out weakly labeled genes (ie, those with a weak signal), we analyzed only strongly labeled genes normalized with GAPDH levels. Genes with a fold difference of $>1.5$ or $<0.5$ were considered significant and were subjected to a validation study, such as real-time quantitative RT-PCR.

To study kinetic gene regulation after UVB exposure, gene clusters were defined with the primary focus on expression at 24 hours after UVB exposure, the time point at which the most significant difference in 8-oxoG formation was observed. ${ }^{7}$ The significant genes expressed at 3 and 24 hours after UVB exposure were categorized into six clusters, as follows, in terms of expression at 3 and 24 hours after UVB exposure: up-regulated and up-regulated (cluster 1), not significant and up-regulated (cluster 2), down-regulated and up-regulated (cluster 3 ), downregulated and down-regulated (cluster 4), not significant and down-regulated (cluster 5), and up-regulated and down-regulated (cluster 6). Pathway analysis for samples exposed to 24 hours of UVB was performed with Microarray Data Analysis Tool software (version 3.2; Filgen, Nagoya, Japan), which uses GenMAPP (http://www. genmapp.org) and WikiPathways (http://wikipathways. org/index.php/WikiPathways) databases. Once a group of statistically significant $(P<0.05)$ pathway genes was formed, gene relationships within pathways were visualized with schematic figures obtained from WikiPathways.

Two-step real-time quantitative RT-PCR using Universal ProbeLibrary (LightCycler 480 System II; Roche, Mannheim, Germany) was performed to confirm microarray results. The following genes were analyzed using real-time PCR: II1b, Vcan, Apod, Saa3, Aif1, Fbn1, Slit2, Mmp2, Tnf (alias Tnfa), and CxCl1 (alias KC). Total RNAs from the back skin of wild-type mice isolated at 24 hours after UVB exposure at $250 \mathrm{~mJ} / \mathrm{cm}^{2}$ were used to generate a standard curve. Each group of RNAs was assayed in duplicate samples by real-time PCR. Expression levels were normalized to those of GAPDH. The primers pairs were designed in the hyaluronan-binding region domain encompassing the intron of Vcan: 5'-TGGGATTGAAGACACTCAGGA-3' as a forward primer in exon 3 and $5^{\prime}$ TGGCTGCCCTGTAGTGAAA-3' as a reverse primer in exon $4 .{ }^{14}$ Statistical differences were determined using an unpaired $t$-test for relative mRNA expression analyzed; $P<0.05$ was considered to be statistically significant.

\section{UVB Irradiation}

Banks of six TL 20W/12RS fluorescent lamps (Philips, Eindhoven, Holland) were used to irradiate the mice with broad-band UVB. These lamps emit a continuous spectrum from 275 to $390 \mathrm{~nm}$, with peak emission at $313 \mathrm{~nm}$; approximately $65 \%$ of that radiation is within the UVB wavelength range. The irradiance was $3.8 \mathrm{~J} / \mathrm{m}^{2} \cdot \mathrm{s}$ for the lamps at a distance of $40 \mathrm{~cm}$, as measured by an UVR305/365D digital radiometer (Tokyo Optical Company; Tokyo Kogaku Kikai KK, Tokyo, Japan). Before the back skin of mice was taken, the backs of the mice were shaved; the mice were then placed $40 \mathrm{~cm}$ below the bank of lamps and were irradiated. For immunohistochemical and immunofluorescence studies, 8-week-old mice were irradiated with UVB at $250 \mathrm{~mJ} / \mathrm{cm}^{2}$, which is the approximate minimal erythema dose for C57BL/6J mice. 


\section{Analysis of UV-Induced Murine Skin Tumors and Sun-Related Human Skin Tumors}

All skin tumors developed in wild-type and Ogg1 knockout mice after chronic exposure to UVB were sampled for immunohistochemical analyses. Tumors were induced by irradiation with broadband UVB at the minimal erythema dose three times per week for 40 weeks. ${ }^{7,15}$ The histologically examined wild-type samples were squamous cell carcinoma (SCC; $n=5)$, sarcoma $(n=1)$, and papilloma $(n=5)$; the Ogg1 knockout samples were SCC $(n=19)$, sarcoma $(n=4)$, and papilloma $(n=3)$. Human skin tumor samples were also examined for immunohistochemical analysis. Patient samples previously examined by independent dermatopathologists were selected from the archival tissue sample collection of the Division of Dermatology of the Kobe University of Medicine. We focused primarily on human UV-induced malignant and benign tumors. The tumors selected (except for malignant melanoma, all the tumors had developed on sunexposed skin areas) were as follows: malignant melanoma ( $n=32)$, lentigo maligna melanoma $(n=11)$, superficial spreading melanoma $(n=7)$, acral lentiginous melanoma $(n=11)$, nodular melanoma $(n=3)$, basal cell carcinoma ( $n=30)$, SCC $(n=27)$, and actinic keratosis $(n=25)$. In addition, seborrheic keratosis $(n=25)$ and lentigo senilis $(n=18)$ lesions were selected as the benign counterparts.

\section{Histology and Immunohistochemistry}

For histological analyses, the back skin from wild-type and Ogg1 knockout mice, dissected at each time point, was fixed in neutralized $10 \%$ formalin and embedded in paraffin. Then, 4- $\mu \mathrm{m}$ sections were cut, deparaffinized, rehydrated, and washed in PBS. Sections placed in 10 $\mathrm{mmol} / \mathrm{L}$ of citrate buffer ( $\mathrm{pH}$ 6.0) were microwaved three times for 5 minutes. After blocking of endogenous peroxidase, nonspecific binding sites were blocked by incubating the sections with protein blocking serum (Dako, Kyoto, Japan). Sections were incubated for 16 hours at $4^{\circ} \mathrm{C}$ with the following primary antibodies: rabbit polyclonal anti-mouse IL-1 $\beta$ (1:1000 dilution; Abcam, Cambridge, MA), rabbit polyclonal anti-mouse versican (1: 100 dilution; LifeSpan Biosciences, Seattle, WA), rabbit polyclonal anti-human versican (1:125 dilution; Atlas Antibodies, Stockholm, Sweden), or rabbit polyclonal antimouse p53 (CM5) (1:500 dilution; Leica Biosystems Newcastle, Newcastle upon Tyne, UK). The antibody of murine versican that is capable of detecting glycosaminoglycan domain $\beta$ could identify $V 0$ and V1 isoforms, which have been shown to be functional in cell proliferation and anti-apoptosis. ${ }^{16}$ Human versican antibody can be used to detect hyaluronan-binding domain; it was used to detect the V0, V1, V2, and V3 isoforms. After a washing with PBS, the sections were incubated with biotin-conjugated anti-rabbit IgG (IgG; Dako) for 20 minutes at room temperature, followed by incubation for 15 minutes with streptavidin-conjugated horseradish peroxidase (Dako) at room temperature before counterstaining with hematoxylin. Finally, sections were mounted with Glycergel mounting medium (Dako). Samples were analyzed under an all-in-one fluorescence microscope (Biozero BZ-8000; Keyence, Osaka, Japan). For immunofluorescence staining, 4- $\mu \mathrm{m}$ frozen sections were incubated with the rat monoclonal anti-mouse Ly-6G/Gr-1 antibody (1:100 dilution; Pharmingen; BD Biosciences, San Diego, CA) or the rabbit polyclonal anti-mouse versican antibody (1:100 dilution; LifeSpan Biosciences). Then, sections were incubated with Alexa Fluor 488-conjugated secondary antibodies (Invitrogen, Carlsbad, CA) before applying DAPI (Invitrogen). Versican positivity in mouse and human tumors was analyzed and classified as high ( $>50 \%$ of positive cells) or low $(\leq 50 \%)$, diagnosed by two independent dermatopathologists (C.N. and R.O.). To quantify the number of neutrophils present in mouse skin samples after UVB exposure, the number of granulocyte receptor-1 (Gr-1)-positive cells detected by immunofluorescence was counted in ten $800-\mu \mathrm{m}^{2}$ areas. The average number of $\mathrm{Gr}$-1-positive cells was then calculated for each genotype.

Statistical differences were determined using an unpaired $t$-test for the number of Gr-1 positive cells analyzed; $P<0.05$ was considered to be statistically significant.

\section{Western Blot Analysis}

Western blotting was performed as described previously. ${ }^{17}$ Briefly, $10.0 \mu \mathrm{g}$ of protein was electrophoresed on $10 \%$ sodium dodecylsulfate-polyacrylamide gels and transferred to polyvinylidene difluoride membranes. Membranes were incubated with rabbit polyclonal antimouse versican (1:1000 dilution; LifeSpan Biosciences), rabbit polyclonal anti-mouse p53 (CM5) (1:2000 dilution; Leica Biosystems Newcastle), or $\alpha / \beta$-tubulin (1:500 dilution; Cell Signaling Technology, Danvers, MA) as loading control, followed by reaction with alkaline phosphataselinked IgG (Promega, Madison, WI). Immunoreactive bands were visualized after reaction with Nitro Blue Tetrazolium/5-bromo-4-chloro-3'-indole phosphate (NBT/ BCIP) solution (Promega).

\section{Results}

\section{Time-Course Analysis of Gene Expression in Ogg1 Knockout Mice after UVB Exposure}

To investigate which genes are up- or down-regulated after UVB irradiation in wild-type and Ogg1 knockout mice, we performed microarray gene profiling of skin at 3 and 24 hours after exposure. We extracted the significant genes at each time point and sorted the genes with a fold difference of $>1.5$ or $<0.5$, after excluding low-intensity signals. This microarray study revealed that 255 genes were up-regulated and 426 genes were down-regulated in Ogg1 knockout mice at 3 hours after UVB exposure and that 372 and 206 genes were up- and down-regulated at 24 hours after UVB exposure, respectively (Figure 1A). To understand the kinetics of gene regulation 
A

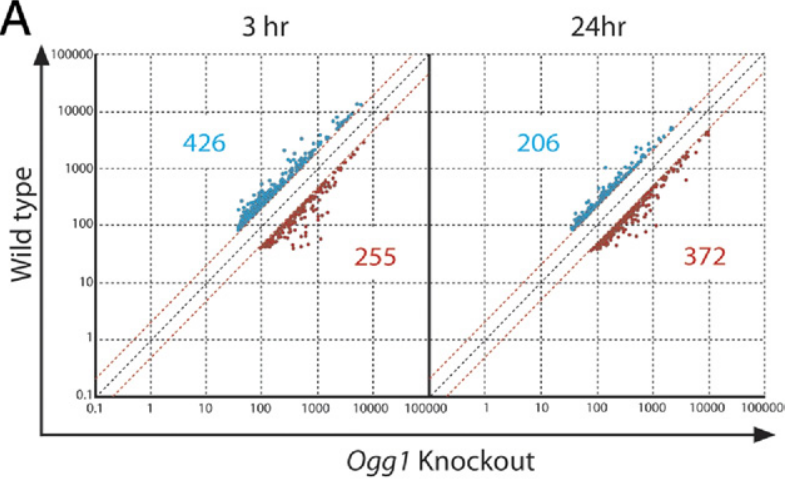

The number of genes

282

68

1

184

21

Figure 1. Time-course analysis of gene expression in $\operatorname{Ogg} 1$ knockout mouse skin after UVB exposure. A: Genes up- or down-regulated at 3 and 24 hours after exposure of $\operatorname{Ogg} 1$ knockout and wild-type mice to UVB. Dots represent genes with an expression difference of $>1.5$ or $<0.5$ times (significant genes) after exclusion of low-intensity microarray signals. Red and blue dots represent up- and down-regulated genes in Ogg1-knockout, respectively, with the number of up- and down-regulated genes indicated in the corresponding color. B: Clusters of up- and down-regulated genes at each time point were categorized based on the trend of up- or down-regulation at 3 and 24 hours after UVB exposure. Schematics are shown for the six clusters, along with the number of genes assigned to each cluster.

after UVB exposure, gene clusters were defined with a primary focus on expression at 24 hours after UVB exposure, at which point the most significant difference in 8-oxoG formation was observed. ${ }^{7}$ The main reason for using cluster analysis in the present study was to identify the constitutively up-regulated (down-regulated) genes because we speculated that among those genes, there may be the most probable candidate genes for skin cancers with positive (negative) driving forces by up-regulated (down-regulated) genes. The numbers of significant genes in each cluster at 3 and 24 hours after UVB exposure are presented in Figure 1B. These data were further analyzed in our next study.

\section{The Inflammatory Pathway Is the Most Affected among Up-Regulated Genes at 24 Hours after UVB Exposure}

Previously, we have shown by immunohistochemistry that UVB-induced epidermal 8-oxoG accumulation is significantly different between wild-type and Ogg1 knockout

mice at 24 hours, compared with 3 hours, after UVB exposure. ${ }^{7}$ First, to elucidate gene regulation in terms of the pathways affected in Ogg1-knockout mice after UVB, we performed pathway analysis using the Microarray Data Analysis Tool (GenMAPP and WikiPathways) for 372 up-regulated and 206 down-regulated genes in Ogg1knockout at 24 hours after UVB exposure. We selected significant pathways with high $Z$-score and low $P$ value $(<0.5)$. Surprisingly, the inflammatory response pathway was the most significantly affected pathway, with 7 of 39 genes altered (Table 1). The second most significant pathway was the complement and coagulation cascade. We further investigated the significant genes involved in the inflammatory pathway. Those genes included $/ / 1 \mathrm{~b}$, Cd80, Col1a2, Col3a1, Fn1, Fcgr1, and Ighm (alias Igh-6). The pathway involvement of seven genes previously shown to be related to the skin is presented in Figure 2A.

We selected $/ 11 b$, which is one of the key genes expressed by macrophages, for validation studies. Using realtime quantitative RT-PCR, we confirmed that the expression levels of $/ 11 \mathrm{~b}$ in Ogg1 knockout mice at 24 hours after UVB exposure were 7.1-fold higher than in the wild-type counterparts (Figure 2B). Moreover, we studied the expression pattern of IL-1 $\beta$ in the skin of UVB-exposed or control Ogg1 knockout mice. Expression of $\mathrm{IL}-1 \beta$ in the epidermis and dermal cells in Ogg1 knockout mice was higher than in the wild-type mice. On the other hand, nonirradiated skin was negative for $\mathrm{IL}-1 \beta$ (Figure $2 \mathrm{C}$ ), which is consistent with results from the microarray and real-time PCR analyses.

\section{Versican Is Strongly Up-Regulated in the Skin and in UVB-Induced Tumors of Ogg1 Knockout Mice}

Accumulation of 8-oxoG in the epidermis at 3 and 24 hours after UVB exposure is higher in Ogg1 knockout mice than in wild-type mice, implying that the continuously up- or down-regulated genes are involved in skin tumor development in Ogg1 knockout mice. ${ }^{7}$ Next, we focused on constitutively up-regulated (or down-regu-

Table 1. Pathway Analysis for Changes in Gene Expression in Ogg $1^{-/}$Mice 24 Hours after UVB Exposure

\begin{tabular}{cccc}
\hline & $\begin{array}{c}\text { Total } \\
\text { genes } \\
\text { Pathway name* }\end{array}$ & $\begin{array}{c}\text { Changed } \\
\text { expression } \\
\text { (no.) }\end{array}$ & P value \\
\hline Increased Expression & & & \\
$\quad$ Inflammatory response & 39 & 7 & 0.000054 \\
$\quad$ Complement/coagulation & 56 & 7 & 0.00042 \\
Striated muscle contraction & 41 & 5 & 0.0032 \\
$\quad \begin{array}{c}\text { Complement activation } \\
\quad \text { (classical pathway) }\end{array}$ & 13 & 3 & 0.0049 \\
$\begin{array}{c}\text { Decreased Expression } \\
\text { Hypertrophy model }\end{array}$ & 19 & 3 & 0.0015 \\
$\quad$ GPCR (class A, & 187 & 7 & 0.0035 \\
$\quad$ rhodopsin-like) & & & \\
Id signaling pathway & 48 & 3 & 0.016 \\
Small ligand GPCRs & 19 & 2 & 0.020 \\
\hline
\end{tabular}

*Top four pathways involving significantly increased or decreased gene expression, as extracted from microarray data.

GPCR, G protein-coupled receptor. 
A

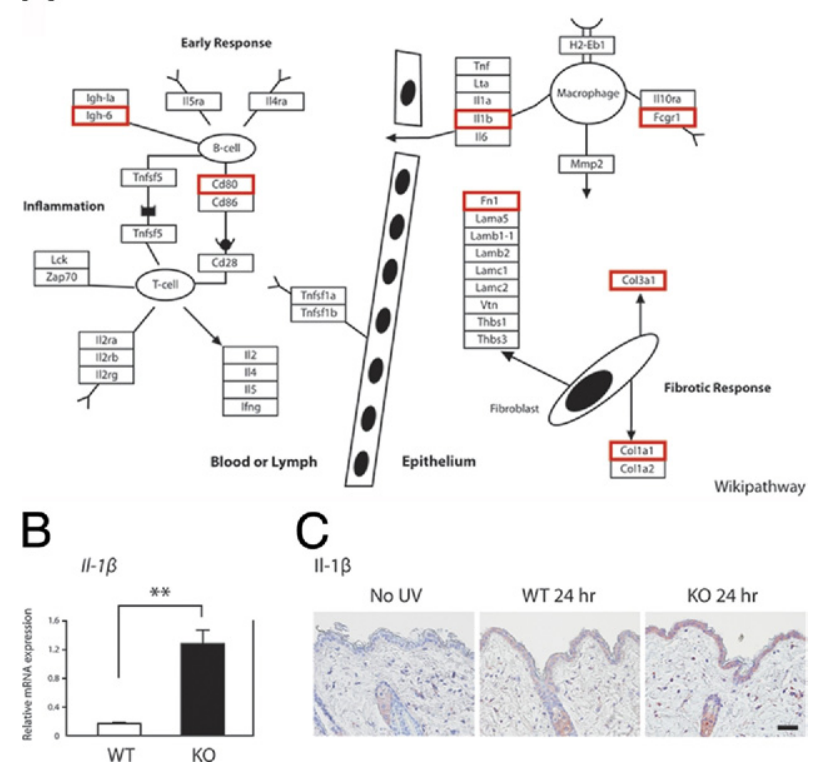

Figure 2. The inflammatory pathway is the most affected among the upregulated genes at 24 hours after UVB exposure. A: The relationships among significantly up-regulated genes involved in inflammatory response pathways in Ogg 1 knockout mice at 24 hours after UVB exposure. Significantly up-regulated genes, previously shown to be involved in skin, were selected from microarray data (red rectangles). The schematic figure was modified from WikiPathways (available at http://www.wikipathways.org/index.php Pathway: WP458). B: Relative IL- $1 \beta$ gene expression levels in wild-type (WT) and Ogg 1 knockout (KO) mouse skin, as determined by real-time quantitative PCR. Each of the two groups of RNAs isolated from wild-type and $O g g 1$ knockout mice was assayed in duplicate. ${ }^{* * *} P<0.01$. Data are representative of three separate determinations. C: IL- $1 \beta$ gene expression levels in the skin of wild-type and Ogg 1 knockout mice at 24 hours after UVB exposure. Positive signals are seen as reddish-brown deposits produced on reaction with the 3-amino-9-ethylcarbazole substrate. The UVB dose was $250 \mathrm{~mJ} / \mathrm{cm}^{2}$. Weak or no expression of IL- $1 \beta$ is seen in wild-type mouse skin with and without UVB exposure. Scale bar $=30 \mu \mathrm{m}$.

lated) genes, because we speculated that some of these genes are the most probable candidate genes for developing skin cancers with correspondingly positive or negative driving forces of the up-regulated or down-regulated) genes. Of note, 22 continuously up-regulated genes (cluster 1) were observed in gene clusters, but only one gene was continuously down-regulated (cluster 4) (Figure 1B). These data suggest that some of the 22 continuously up-regulated genes in UVB-exposed Ogg1 knockout mice are likely to contribute to skin carcinogenesis. These 22 genes are detailed in Table 2 .

We performed validation studies for some of the 22 genes by real-time quantitative RT-PCR. Consistent with the microarray data, the expressions of Vcan, Saa3, Fbn1, and Slit2 were significantly higher in Ogg1 knockout mice than in wild-type mice, both at 3 and at 24 hours after UVB exposure (Figure 3A). Again, in agreement with the microarray data, the expression levels of Apod and Aif1 in Ogg1 knockout mice were nearly twice of those in wildtype mice (see Supplemental Figure S1 at http://ajp. amjpathol.org). Of the six genes analyzed by RT-PCR, versican (Vcan) expression most significantly differed between the wild-type and knockout mice; knockout levels were 2.9- and 7.2-fold higher than wild-type at 3 and 24 hours after UVB exposure, respectively. Versican is widely expressed in the $\operatorname{skin}^{18}$ and has been implicated in a wide variety of biological functions during tumorigenesis. ${ }^{11,19,20}$ We therefore focused on versican to further dissect the UVB-induced skin carcinogenic phenotype of Ogg1 knockout mice.

To determine the expression pattern of versican in the skin after UVB exposure, we performed immunohistochemical staining for versican on the skin of control and UVB-irradiated (24 hours) wild-type and Ogg1 knockout mice. In wild-type mice, versican was strongly expressed in the epidermis after UVB exposure, but was barely expressed in nonexposed skin (Figure 3B). On the other hand, versican expression was markedly stronger in Ogg1 knockout mice at 24 hours after UVB exposure than the wild-type counterparts; this strong expression was observed not only in the epidermis but also in some dermal components, such as fibroblasts (Figure 3B). We also performed Western blot analysis for versican of the wild-type and Ogg1 knockout mouse skin after UVB exposure and found that versican was more up-regulated in the knockout mice than in the wild-type mice at both 24

Table 2. Increased Expression of 22 Genes in $\operatorname{Ogg} 1^{-1-}$ Mice at 3 and 24 Hours after UVB Exposure

\begin{tabular}{|c|c|c|}
\hline & Gene symbol & Gene name \\
\hline 1 & Bst2 & Bone marrow stromal cell antigen 2 \\
\hline 2 & $C f b$ & Complement factor B \\
\hline 3 & $\mathrm{Cxcl13}$ & Chemokine (C-X-C motif) ligand 13 \\
\hline 4 & Apod & Apolipoprotein D \\
\hline 5 & & $\begin{array}{l}\text { Mus musculus hemoglobin } \mathrm{Y} \text {, beta- } \\
\text { like embryonic chain (Hbb-y), } \\
\text { mRNA }\end{array}$ \\
\hline 6 & Ms4a6c & $\begin{array}{l}\text { Membrane-spanning 4-domains, } \\
\text { subfamily } A \text {, member } 6 C\end{array}$ \\
\hline 7 & & $\begin{array}{l}\text { Mus musculus Fc receptor-like } \\
\text { protein } 2 \text { immunoglobulin short } \\
\text { Isoform (Fcrh2) mRNA, complete } \\
\text { cds }\end{array}$ \\
\hline 8 & & $\begin{array}{l}\text { Maa26e10.y1 NCI_CGAP_Li10 Mus } \\
\text { musculus cDNA clone } \\
\text { IMAGE:3812083 } 5\end{array}$ \\
\hline 9 & Tyrobp & $\begin{array}{l}\text { TYRO protein tyrosine kinase } \\
\text { binding protein }\end{array}$ \\
\hline 10 & Vcan & Versican \\
\hline 11 & Saa3 & Serum amyloid A 3 \\
\hline 12 & 9530053H05Rik & RIKEN cDNA 9530053H05 gene \\
\hline 13 & Aif1 & Allograft inflammatory factor 1 \\
\hline 14 & & $\begin{array}{l}\text { Mus musculus retinoic acid } \\
\text { receptor responder (tazarotene } \\
\text { induced) } 2 \\
\text { (Rarres2), mRNA }\end{array}$ \\
\hline 15 & Fcer1g & $\begin{array}{l}\text { Fc receptor, IgE, high affinity } 1 \text {, } \\
\text { gamma polypeptide }\end{array}$ \\
\hline 16 & & $\begin{array}{l}\text { BY765147 RIKEN full-length } \\
\text { enriched, activated spleen Mus } \\
\text { musculus }\end{array}$ \\
\hline & & $\begin{array}{l}\text { cDNA clone F830013C14 3-, } \\
\text { mRNA sequence }\end{array}$ \\
\hline 17 & Pth1r & Parathyroid hormone 1 receptor \\
\hline 18 & Fbn1 & Fibrillin 1 \\
\hline 19 & Slit2 & Slit homolog 2 (Drosophila) \\
\hline 20 & Gpx7 & Glutathione peroxidase 7 \\
\hline 21 & Cpxm1 & Carboxypeptidase X 1 (M14 family) \\
\hline 22 & Pf4 & Platelet factor 4 \\
\hline
\end{tabular}

Increased expression was determined on the basis of a ratio of $\mathrm{Ogg} 1$ wild-type to knockout being $>2.0$, as determined from the microarray data at each time point. 
A
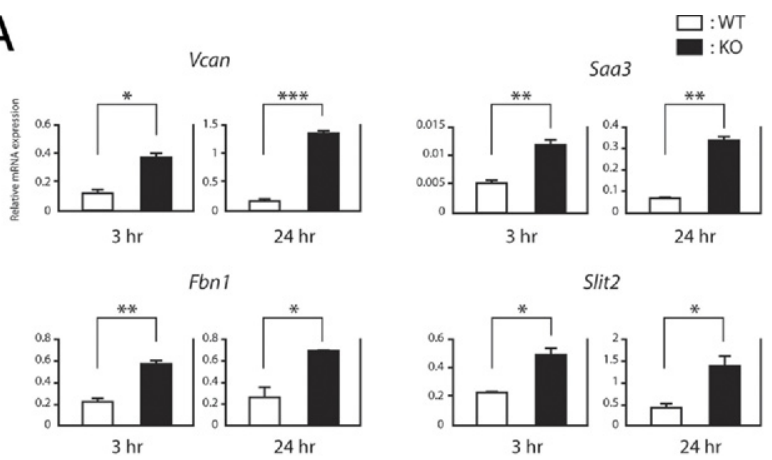

B Versican
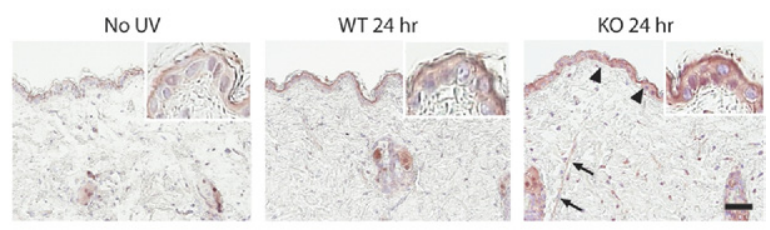

C

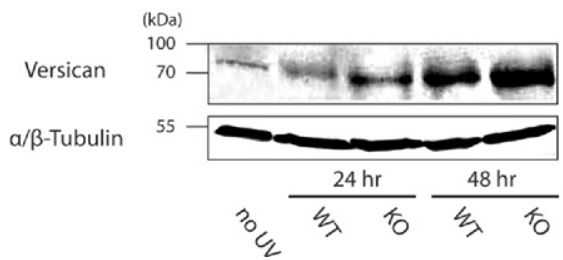

Figure 3. Expression of continuously up-regulated genes after UVB exposure. A: Four genes were significantly up-regulated at both 3 and 24 hours after UVB exposure. Relative expression levels were determined by real-time quantitative PCR. Each of the two groups of RNAs isolated from wild-type and Ogg 1 knockout mice was assayed in duplicate. Reactions were normalized to GAPDH expression levels. ${ }^{*} P<0.05$; ${ }^{* *} P<0.01$; and ${ }^{* * * *} P<0.001$. B: Immunohistochemical study of versican expression after UVB irradiation. Versican is barely expressed in the wild-type mouse epidermis and dermis in the absence of UVB irradiation. At 24 hours after UVB exposure, versican was expressed in the wild-type epidermis. In Ogg1 knockout mice at 24 hours, versican was strongly expressed in the epidermis (arrowheads), as well as in dermal fibroblasts (arrows). Positive signals are seen as reddish-brown deposits produced on reaction with the 3-amino-9-ethylcarbazole substrate. Scale bar $=30 \mu \mathrm{m}$. C: Versican expression after UVB exposure, as determined by Western blotting. Versican was more strongly up-regulated in Ogg 1 knockout than in wild-type mice at 24 and 48 hours after UVB exposure. This up-regulation was time-dependent. The band at approximately $75 \mathrm{kDa}$ indicates that the antibody for versican can detect the $\mathrm{V} 1$ isoform. $\alpha / \beta$-Tubulin was used as the loading control. Data are representative of three separate determinations.

and 48 hours after UVB exposure. In addition, we found that the expression levels of versican indicating $\mathrm{V} 1$ isoform were up-regulated in a time-dependent manner (Figure 3C).

\section{Versican Is Strongly Expressed in Skin Tumors Induced in Ogg1 Knockout Mice after Long-Term UVB Exposure}

We found that high versican expression after single UVB irradiation is associated with Ogg1 gene disruption. We then investigated whether differences in versican expression between wild-type and Ogg1 knockout mice can also be found in skin tumors induced by long-term UVB irradiation. We irradiated wild-type and Ogg1 knockout mice with UVB for more than 40 weeks and obtained 11 tumors in the wild-type mice and 26 tumors in the knockout mice; we diagnosed these tumors histologically as SCC, sarcoma, and papilloma. ${ }^{7}$ We performed immunohistochemical staining for versican in all these tumors. Versican was expressed in 17 of 19 SCCs in the knockout mice and in 2 of 5 SCCs in the wild-type mice (Figure 4). All the sarcomas (one in the wild-type and four in the knockout mice) were strongly positive for versican. However, only one of five papillomas in the wild-type and one of three papillomas in the knockout mice were highly positive for versican $(P<$ 0.05 for the ratio of malignant tumor/analyzed total tumors of each genotype). Thus, versican was more highly expressed in Ogg1 knockout mouse tumors than in the wild type. In conclusion, versican expression is especially evident in malignant tumors, compared with benign skin tumors.

\section{Versican Is Expressed in Various Sun-Related Human Skin Tumors}

To further examine versican expression, we investigated whether versican is expressed in human skin tumors associated with long-term UV exposure. We performed immunohistochemistry in 157 human skin tumors. We selected tumors that had developed on sun-exposed areas of the skin (the exception being malignant melanoma). Strong versican expression was seen mostly in the dermis and in the stromal components around the tumors, rather than in the tumor cells (Figure 5, A and B). This observation was more evident in malignant than in benign tumors. Basal cell carcinoma, for instance, was positive for versican in $80 \%$ (24/30) of the analyzed tumors; expression was noted in stromal areas around the tumor cells (Figure 5A). Lentigo maligna melanoma, a malignant melanoma that usually develops on chronically sun-exposed areas, revealed strong versican expression in the dermis of 8 of 11 tumors. The other three malignant melanoma types showed milder versican expression. These data suggest that lentigo maligna melanoma might be strongly associated with UV/ ROS-induced 8-OxOG formation and that it might be of different etiology as opposed to the other malignant mela-

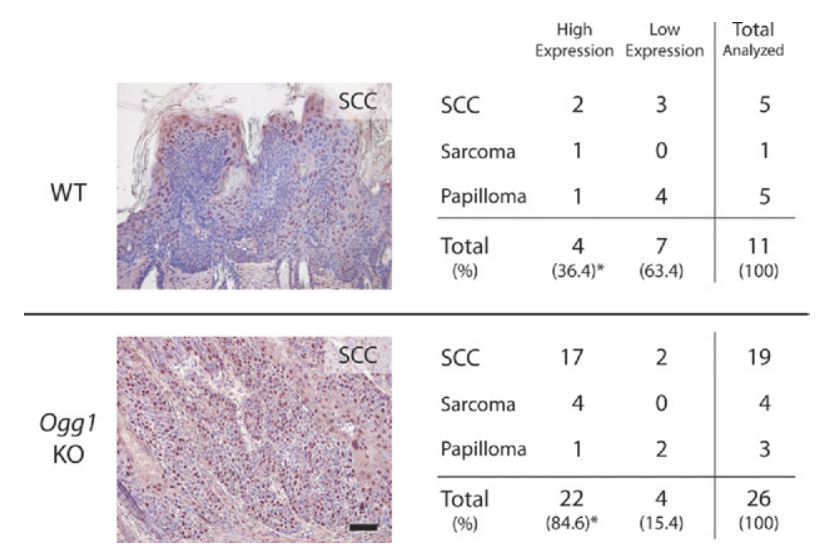

Figure 4. Versican expression in developing skin tumors of chronically UVB-exposed mice. Representative histological sections of SCC tumors from wild-type and Ogg1 knockout mice red-colored staining positively for versican are shown, with a summary of versican-positive wild-type and Ogg1 knockout mouse tumors overall. ${ }^{*} P<0.05$ for the ratio of malignant tumor to total tumors analyzed for each genotype. 

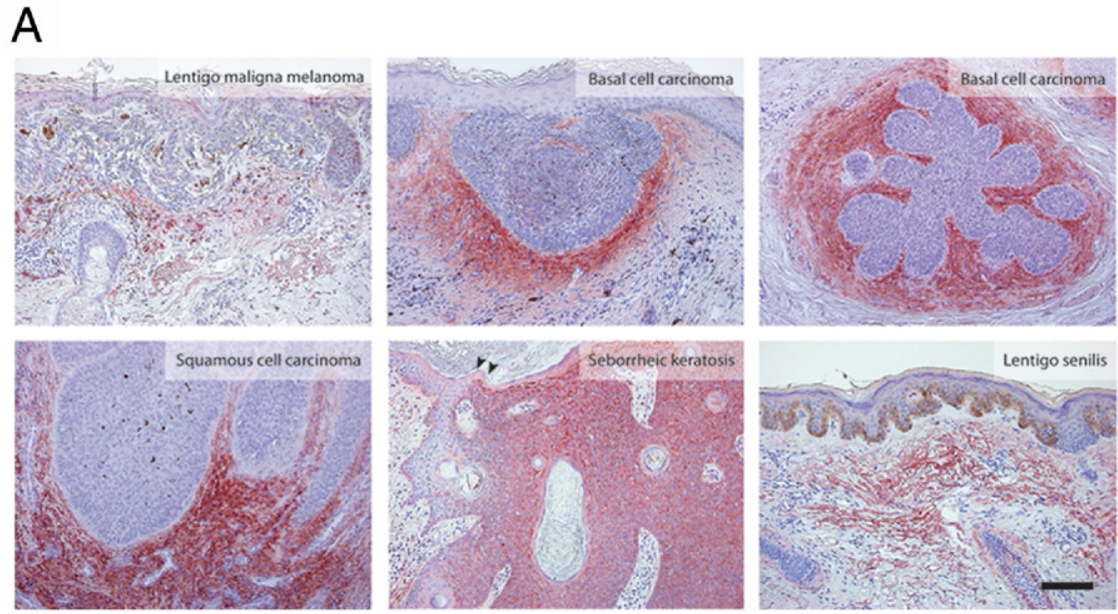

B

\begin{tabular}{|c|c|c|c|c|c|}
\hline \multirow{3}{*}{ Human skin tumors } & \multirow{3}{*}{$\begin{array}{l}\text { The number } \\
\text { of Patients }\end{array}$} & \multicolumn{4}{|c|}{ Versican expression } \\
\hline & & \multicolumn{2}{|c|}{ Dermal and stromal components } & \multicolumn{2}{|c|}{ Tumor cells } \\
\hline & & $\begin{array}{c}\text { High } \\
\text { Expression }\end{array}$ & $\begin{array}{c}\text { Low } \\
\text { Expression }\end{array}$ & $\begin{array}{c}\text { High } \\
\text { Expression }\end{array}$ & $\begin{array}{l}\text { Low } \\
\text { Expression }\end{array}$ \\
\hline Malignant & 114 & 51 & 63 & 11 & 103 \\
\hline Malignant melanoma & 32 & 9 & 23 & 4 & 28 \\
\hline Lentigo maligna melanoma & 11 & 7 & 4 & 2 & 9 \\
\hline Superficial spreading melanoma & 7 & 1 & 6 & 1 & 6 \\
\hline Acral lentiginous melanoma & 11 & 1 & 10 & 1 & 10 \\
\hline Nodular melanoma & 3 & 0 & 3 & 0 & 3 \\
\hline Basal cell carcinoma & 30 & 22 & 8 & 6 & 24 \\
\hline Squamous cell carcinoma & 27 & 15 & 12 & 1 & 26 \\
\hline Actinic keratosis & 25 & 5 & 20 & 0 & 25 \\
\hline Benign & 43 & 14 & 29 & 3 & 40 \\
\hline Seborrheic keratosis & 25 & 6 & 19 & 3 & 22 \\
\hline Lentigo senilis & 18 & 8 & 10 & 0 & 18 \\
\hline
\end{tabular}

Figure 5. Versican expression in human skin tumors. A: Immunohistochemical study of versican expression in malignant (lentigo maligna melanoma, basal cell carcinoma, and squamous cell carcinoma) and benign (seborrheic keratosis and lentigo senilis) tumors. Arrowheads indicate the borders between the seborrheic keratosis tumor and the normal skin. Scale bar $=100 \mu \mathrm{m}$. B: Classification of versican expression in skin tumors, grouped as dermal and stromal versus tumoral.

noma types. Although versican expression was relatively weaker in benign tumors than in malignant skin tumors, some tumors, such as seborrheic keratosis, showed stronger expression in tumor cells than in the dermis around the tumors (Figure 5, A and B).

\section{High Versican Expression Is Accompanied by Inflammatory Neutrophil Infiltration}

Finally, we investigated the mechanisms associated with UVB-induced high versican expression in Ogg1 knockout mice. Notably, we found that most human malignant and benign tumors with high versican expression were enriched with inflammatory cells in the tumors or in the dermis around them (Figure 6A). Versican was highly expressed both in inflammatory cells, especially segmented leukocytes (neutrophils), and in the dermal component. To ascertain whether dermal neutrophils interact with versican, we performed immunofluorescence double staining for versican and the neutrophil marker Gr-1. We irradiated wild-type mice with a single minimal erythema dose of UVB and dissected the skin after 24 hours. Gr-1 and versican were colocalized in infiltrated neutrophils, indicat- ing that UVB-induced skin neutrophils interact with versican (Figure 6B). The interaction between versican and neutrophils suggests that the amount of neutrophils differs between wild-type and Ogg1 knockout mice. Indeed, when we compared the number of neutrophils infiltrated in the dermis at 24 and 48 hours after UVB exposure between wild-type and Ogg1 knockout mice, ${ }^{20}$ the number of neutrophils was significantly increased in Ogg1 knockout mice, compared with the wild-type mice (Figure 6C). Taken together, high versican expression in Ogg1 knockout mouse skin after UVB exposure is highly associated with the inflammatory response, particularly neutrophil infiltration (Figure 7).

\section{Discussion}

\section{Gene Profiling of UVB-Irradiated Ogg1 Knockout Mice}

UV rays induce ROS formation, which provoke DNA damage in the skin and subsequent formation of DNA damage products, such as 8 -oxoG. ${ }^{6}$ Previously, we have shown that Ogg1 knockout mice exhibit a highly carcinogenic phenotype, with more early-induced malignant skin 
A

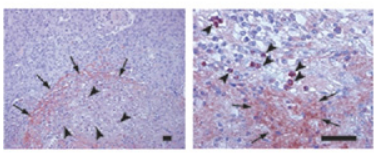

B

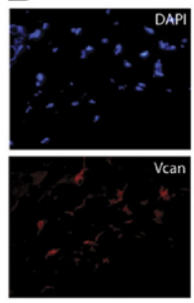

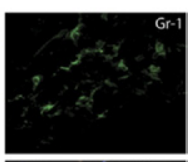

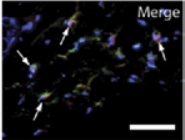

C
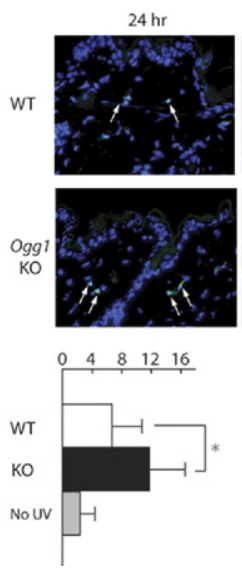

Figure 6. Versican expression and neutrophil localization in human and murine skin tumors. A: Versican expression and inflammatory cells in human squamous cell carcinoma. Versican is strongly expressed in the dermal components (arrows) and inflammatory cells (arrowheads), seen at low magnification (left) and high magnification (right). Two focused areas are taken from different part of sections. Inflammatory cells, especially segmented leukocytes (neutrophils) (arrowheads), were strongly positive for versican. Scale bars $=30 \mu \mathrm{m}$. B: Versican expression and neutrophils in mouse skin. In the merged iamge, neutrophils with versican expression (arrows) appear yellow. Scale bar $=30 \mu \mathrm{m}$. C: Neutrophil infiltration after UVB irradiation in wild-type and Ogg 1 knockout mice. Arrows indicate Gr-1-positive cells (green) in the dermis at 24 and 48 hours after UVB exposure in the wild-type and Ogg1 knockout mice. Scale bar $=30 \mu \mathrm{m}$. The accompanying graphs show the average number of neutrophils per $800 \mu \mathrm{m}^{2}$ at each time point. ${ }^{*} P<0.02$.

tumors than their wild-type counterparts. ${ }^{7}$ Although several gene profiling studies have addressed the UVB response using primary human keratinocytes ${ }^{21}$ or human skin xenografts ${ }^{22}$ or the radiation effects using Ogg1deficient human leukemia cell lines, ${ }^{23}$ the gene pathways in the Ogg1-mediated UVB response remain to be elucidated. We found that the most affected pathway in Ogg1 knockout mice is the inflammatory response pathway. Although the accumulation of DNA damage does not seem to directly influence the inflammatory response, persistent DNA damage response might allow the damaged cells to compromise the biological state of the surrounding tissue, which then secretes inflammatory cytokines. This altered inflammatory state will create a permissive microenvironment that allows the proliferation and metastasis of tumor cells. ${ }^{24,25}$ Rodier et $\mathrm{al}^{26}$ reported that irreparably large radiation doses induce DNA double-strand breaks and increase IL-6 secretion. We compared $I L-6$ levels after $250 \mathrm{~mJ} / \mathrm{cm}^{2}$ of UVB irradiation (approximately 1 minimal erythema dose) between the wild-type and Ogg1 knockout mice by real-time PCR and found that Ogg1 knockout mice express significantly higher levels of IL-6 at 3 and 24 hours after UVB exposure than do wild-type mice (see Supplemental Figure S2 at http://ajp.amjpathol.org). We also performed RT-PCR for inflammatory Mmp2 and Tnf, but found no significant difference between the two Ogg1 genotypes (data not shown).

The present data indicate that $\mathrm{IL}-1 \beta$ and $\mathrm{IL}-6$ are the most important candidate cytokines to induce inflammatory conditions associated with 8-oxoG accumulation after UVB exposure. However, the exact inflammatory pathway genes that are involved in 8-oxoG-induced skin tumorigenesis still remain to be fully elucidated.

\section{Role of Versican in UVB/8-OxoG-Induced Skin Tumorigenesis in Mice and Humans}

Versican is a large chondroitin sulfate proteoglycan. ${ }^{27}$ In the skin, versican is expressed not only in the associated elastic fibers in the dermis but also in the epidermis, hair follicles, and sweat glands. ${ }^{18,28}$ Versican is also known to be expressed in photoaged skin, ${ }^{29}$ which is consistent with our immunohistochemical finding that chronically sun-exposed skin tumors of Ogg1 knockout mice expressed high levels of versican, thus implying that continuous accumulation of 8-oxoG induced by UV leads to versican overexpression and photoaged skin. Versican has also been reported in a wide variety of cancers. ${ }^{16,19}$ Elevated expression of stromal versican predicted increased risk and rate of relapse. ${ }^{30}$ Moreover, versican is highly expressed in the metastatic lesions of pharyngeal

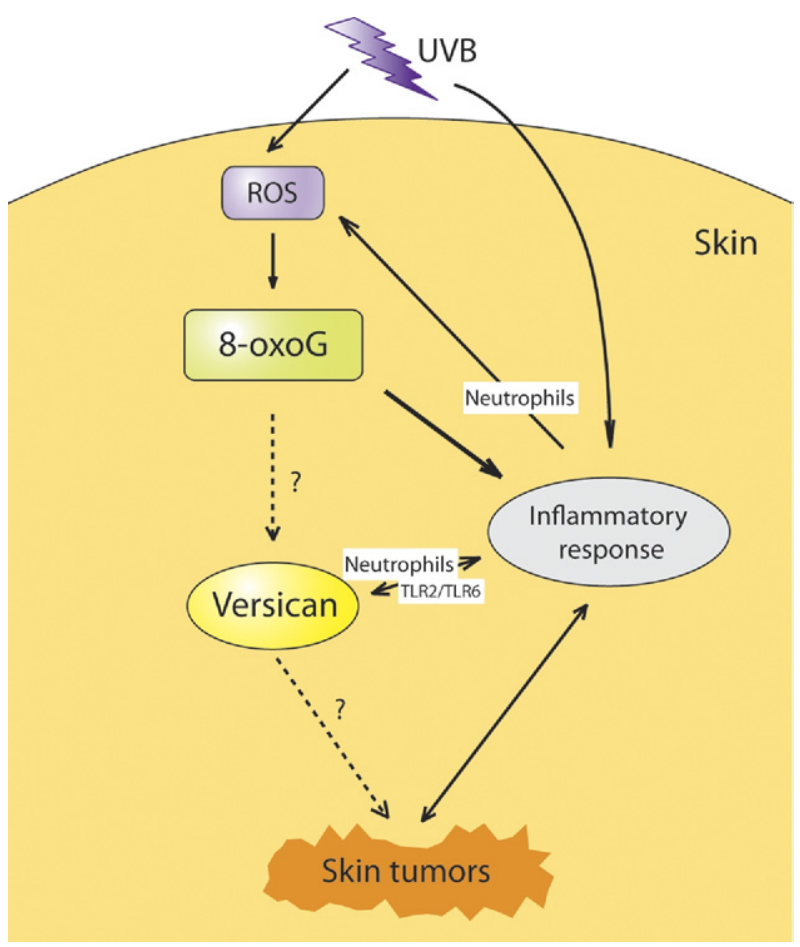

Figure 7. Proposed relationships of versican in the inflammatory response leading to the development of skin tumors in terms of UVB-induced 8-oxoG accumulation. The accumulation of UVB/ROS-induced 8-OxOG in the skin leads to inflammatory reactions. High versican expression is induced by a highly inflammatory microenvironment with high numbers of infiltrated neutrophils; conversely, neutrophil infiltration induces versican overexpression. More ROS will be generated at the inflammatory sites by neutrophils. 
squamous cell carcinoma. ${ }^{31}$ Our immunohistochemistry studies revealed that versican expression is significantly higher in UVB-exposed Ogg1 knockout mice than in the wild-type counterparts. Versican overexpression induces skin tumor development, especially malignant tumors in Ogg1 knockout mice exposed to long-term UVB irradiation. In mouse skin tumors, versican is expressed in not only the dermal stroma but also intracellular components of the epidermis. These observations are in agreement with the results from immunohistochemical studies in the skin at 24 hours after UVB irradiation (Figure 3B). Furthermore, we evaluated human skin tumors related to longterm UV exposure and found that several skin tumors, including malignant melanomas, exhibit high versican expression. These data suggest that human UV-related skin tumors are associated with UV-induced 8-oxoG generation, as well as other DNA damage products previously reported to cause mutation-prone DNA damages by $U V^{2}{ }^{2}$ such as cyclobutane pyrimidine dimers and (6-4) photoproducts.

Among the various types of benign and malignant tumors analyzed in the present study, malignant melanomas exhibited a distinct expression pattern for versican. Of the four malignant melanoma types analyzed (ie, lentigo maligna melanoma, superficial spreading melanoma, acral lentiginous melanoma, and nodular melanoma), lentigo maligna melanoma, which is thought to be strongly associated with chronic UV exposure, showed the strongest versican expression. These results indicate that these four subtypes of malignant melanoma have different pathogenesis in terms of UV induction and 8-oxoG accumulation. Versican was significantly and strongly expressed in basal cell carcinoma, another UV-related skin tumor, especially in the stromal components surrounding the tumors. Although strong versican expression in basal cell carcinoma could be attributed to 8-oxoG accumulation after long-term UV exposure, we consider the possibility that development of basal cell carcinoma is associated with versican up-regulation through epithelial and mesenchymal interactions that occur during hair follicle development. In fact, basal cell carcinoma is a hair follicle-oriented tumor, and the versican promoter is activated during hair follicle development. ${ }^{32}$

\section{Interaction between 8-OxoG and Versican in the Development of Skin Tumors}

Gene profiling of UVB-irradiated Ogg1 knockout mouse skin showed that 8-oxoG accumulation in the skin leads to high inflammatory responses. Furthermore, the highly inflammatory state in Ogg1 knockout mice is accompanied by neutrophil infiltration. Neutrophil infiltration might increase the susceptibility of the epidermal and dermal microenvironment to the development of versican-positive skin cancers, which might be one of the mechanisms for high 8-oxoG accumulation leading to high versican expression in skin tumors (Figure 6). These data are consistent with a previous report showing that a chondroitin sulfate proteoglycan such as versican has a specific receptor for platelet factor 4 (PL-4), a member of the $\alpha$-chemokine subfamily of cytokines on human neutro- phils $^{33}$ (Table 2). Versican carries many strong negatively charged glycosaminoglycans. One of the main glycosaminoglycans, chondroitin sulfate, establishes the interaction between versican and various cytokines and chemokines, ${ }^{34}$ modulating their function. ${ }^{10}$ The interaction of $\mathrm{L}$ - and P-selectins and the CD44-expressing leukocytes, fibroblasts, and epithelial cells with versican also suggests a role of versican in leukocyte infiltration into the ECM. ${ }^{35}$ In addition, P-selectin glycoprotein ligand-1 (PSGL-1), a glycoprotein expressed on the cell surface of neutrophils, binds to the G3 domain of the extracellular proteoglycan versican and mediates leukocyte aggregation, which might be another implication of association between versican and neutrophils. ${ }^{36,37}$

In the present study, the levels of IL-6, a key cytokine triggered by persistent DNA damage, ${ }^{26}$ were significantly up-regulated in Ogg1 knockout mouse skin at both 3 and 24 hours after UVB exposure (see Supplemental Figure S2 at $h$ ttp://ajp.amjpathol.org). Previously, we showed that $\mathrm{CxC} / 1 / \mathrm{KC}$, a chemoreactant gene, is associated with neutrophils during skin inflammation and is more up-regulated when UVB is irradiated..$^{38}$ In the present study, we found no differences in CxC/1/KC expression in the skin of UVB-exposed wild-type and Ogg1 knockout mice, as determined by real-time PCR (data not shown). Recently, it was reported that versican functions as a macrophage activator via the Toll-like receptor family members TLR2 and TLR6, leading to a more inflammatory microenvironment in which cancer cells can be easily grown. ${ }^{25,39} \mathrm{Kim}$ et $\mathrm{al}^{25}$ reported the up-regulation of $1 / 1 \mathrm{~b}$ in metastatic Lewis lung carcinoma and showed that versican functions as a macrophage activator. Our present data indicate that IL-1 $\beta$ and IL-6 are the most important candidate cytokines in inducing inflammation associated with 8-oxoG accumulation after UVB exposure. Versican and the inflammatory response might be reciprocally up-regulated in the skin after UVB exposure. A highly inflammatory microenvironment that is rich in neutrophils will produce more ROS by positive feedback $^{40}$ (Figure 7 ). Although our present data clearly suggest that high versican expression colocalizes with neutrophil infiltrated in UVB-exposed Ogg1 knockout mice skin, the exact cytokine profile associated with those interactions, and whether the accumulation of 8-oxoG up-regulates versican directly after UVB exposure, remain to be elucidated.

In conclusion, the accumulation of UVB/ROS-induced 8-oxoG in the skin leads to inflammatory reactions. A highly inflammatory microenvironment characterized by neutrophil infiltration up-regulates versican expression. Versican overexpression contributes to more aggravated skin inflammation, ultimately leading to the development of skin tumors. The present study provides new clues for understanding the mechanism underlying ROS-induced skin tumorigenesis after UV irradiation.

\section{Acknowledgment}

We thank Dr. Seiji Takeuchi for critical reading of this manuscript and for technical assistance. 


\section{References}

1. Kraemer KH: Sunlight and skin cancer: another link revealed. Proc Natl Acad Sci USA 1997, 94:11-14

2. Nishigori $C$, Hattori $Y$, Toyokuni S: Role of reactive oxygen species in skin carcinogenesis. Antioxid Redox Signal 2004, 6:561-570

3. Maki $H$, Sekiguchi M: MutT protein specifically hydrolyses a potent mutagenic substrate for DNA synthesis. Nature 1992, 355:273-275

4. Sakamoto K, Tominaga Y, Yamauchi K, Nakatsu Y, Sakumi K, Yoshiyama K, Egashira A, Kura S, Yao T, Tsuneyoshi M, Maki H, Nakabeppu Y, Tsuzuki T: MUTYH-null mice are susceptible to spontaneous and oxidative stress induced intestinal tumorigenesis. Cancer Res 2007, 67:6599-6604

5. Tsuzuki T, Nakatsu Y, Nakabeppu Y: Significance of error-avoiding mechanisms for oxidative DNA damage in carcinogenesis. Cancer Sci 2007, 98:465-470

6. Hattori Y, Nishigori C, Tanaka T, Uchida K, Nikaido O, Osawa T, Hiai $\mathrm{H}$, Imamura S, Toyokuni S: 8-hydroxy-2'-deoxyguanosine is increased in epidermal cells of hairless mice after chronic ultraviolet $B$ exposure. J Invest Dermatol 1996, 107:733-737

7. Kunisada M, Sakumi K, Tominaga Y, Budiyanto A, Ueda M, Ichihashi M, Nakabeppu Y, Nishigori C: 8-Oxoguanine formation induced by chronic UVB exposure makes Ogg1 knockout mice susceptible to skin carcinogenesis. Cancer Res 2005, 65:6006-6010

8. Nishigori C: Cellular aspects of photocarcinogenesis. Photochem Photobiol Sci 2006, 5:208-214

9. Aburatani H, Hippo Y, Ishida T, Takashima R, Matsuba C, Kodama T, Takao M, Yasui A, Yamamoto K, Asano M: Cloning and characterization of mammalian 8-hydroxyguanine-specific DNA glycosylase/ apurinic, apyrimidinic lyase, a functional mutM homologue. Cancer Res 1997, 57:2151-2156

10. Wu YJ, La Pierre DP, Wu J, Yee AJ, Yang BB: The interaction of versican with its binding partners. Cell Res 2005, 15:483-494

11. Wight TN: Versican: a versatile extracellular matrix proteoglycan in cell biology. Curr Opin Cell Biol 2002, 14:617-623

12. Sakumi K, Tominaga $Y$, Furuichi M, Xu P, Tsuzuki T, Sekiguchi M, Nakabeppu Y: Ogg1 knockout-associated lung tumorigenesis and its suppression by Mth1 gene disruption. Cancer Res 2003, 63:902-905

13. Cui CY, Smith JA, Schlessinger D, Chan CC: X-linked anhidrotic ectodermal dysplasia disruption yields a mouse model for ocular surface disease and resultant blindness. Am J Pathol 2005, 167: 89-95

14. Naso MF, Zimmermann DR, lozzo RV: Characterization of the complete genomic structure of the human versican gene and functional analysis of its promoter. J Biol Chem 1994, 269:32999-33008

15. Kunisada M, Kumimoto $H$, Ishizaki K, Sakumi K, Nakabeppu $Y$, Nishigori C: Narrow-band UVB induces more carcinogenic skin tumors than broad-band UVB through the formation of cyclobutane pyrimidine dimer. J Invest Dermatol 2007, 127:2865-2871

16. Ricciardelli C, Sakko AJ, Ween MP, Russell DL, Horsfall DJ: The biological role and regulation of versican levels in cancer. Cancer Metastasis Rev 2009, 28:233-245

17. Kunisada M, Budiyanto A, Bito T, Nishigori C, Ueda M: Retinoic acid suppresses telomerase activity in HSC-1 human cutaneous squamous cell carcinoma. Br J Dermatol 2005, 152:435-443

18. Zimmermann DR, Dours-Zimmermann MT, Schubert M, BrucknerTuderman L: Versican is expressed in the proliferating zone in the epidermis and in association with the elastic network of the dermis. J Cell Biol 1994, 124:817-825

19. Touab M, Villena J, Barranco C, Arumi-Uria M, Bassols A: Versican is differentially expressed in human melanoma and may play a role in tumor development. Am J Pathol 2002, 160:549-557

20. Lu YP, Lou YR, Yen P, Mitchell D, Huang MT, Conney AH: Time course for early adaptive responses to ultraviolet $B$ light in the epidermis of SKH-1 mice. Cancer Res 1999, 59:4591-4602
21. Sesto A, Navarro M, Burslem F, Jorcano JL: Analysis of the ultraviolet $B$ response in primary human keratinocytes using oligonucleotide microarrays. Proc Natl Acad Sci USA 2002, 99:2965-2970

22. Hachiya A, Sriwiriyanont P, Fujimura T, Ohuchi A, Kitahara T, Takema Y, Kitzmiller WJ, Visscher MO, Tsuboi R, Boissy RE: Mechanistic effects of long-term ultraviolet B irradiation induce epidermal and dermal changes in human skin xenografts. Am J Pathol 2009, 174: 401-413

23. Chaudhry MA: Radiation-induced gene expression profile of human cells deficient in 8-hydroxy-2'-deoxyguanine glycosylase. Int J Cancer 2006, 118:633-642

24. Campisi J: Senescent cells, tumor suppression, and organismal aging: good citizens, bad neighbors. Cell 2005, 120:513-522

25. Kim S, Takahashi H, Lin WW, Descargues P, Grivennikov S, Kim Y, Luo JL, Karin M: Carcinoma-produced factors activate myeloid cells through TLR2 to stimulate metastasis. Nature 2009, 457:102-106

26. Rodier F, Coppe JP, Patil CK, Hoeijmakers WA, Munoz DP, Raza SR, Freund A, Campeau E, Davalos AR, Campisi J: Persistent DNA damage signalling triggers senescence-associated inflammatory cytokine secretion. Nat Cell Biol 2009, 11:973-979

27. Zimmermann DR, Ruoslahti E: Multiple domains of the large fibroblast proteoglycan, versican. EMBO J 1989, 8:2975-2981

28. Bode-Lesniewska B, Dours-Zimmermann MT, Odermatt BF, Briner J, Heitz PU, Zimmermann DR: Distribution of the large aggregating proteoglycan versican in adult human tissues. J Histochem Cytochem 1996, 44:303-312

29. Knott A, Reuschlein K, Lucius R, Stab F, Wenck H, Gallinat S: Deregulation of versican and elastin binding protein in solar elastosis. Biogerontology 2009, 10:181-190

30. Suwiwat S, Ricciardelli C, Tammi R, Tammi M, Auvinen P, Kosma VM LeBaron RG, Raymond WA, Tilley WD, Horsfall DJ: Expression of extracellular matrix components versican, chondroitin sulfate, tenascin, and hyaluronan, and their association with disease outcome in node-negative breast cancer. Clin Cancer Res 2004, 10:2491-2498

31. Pukkila MJ, Kosunen AS, Virtaniemi JA, Kumpulainen EJ, Johansson RT, Kellokoski JK, Nuutinen J, Kosma VM: Versican expression in pharyngeal squamous cell carcinoma: an immunohistochemical study. J Clin Pathol 2004, 57:735-739

32. Kishimoto J, Ehama R, Wu L, Jiang S, Jiang N, Burgeson RE: Selective activation of the versican promoter by epithelial- mesenchymal interactions during hair follicle development. Proc Natl Acad Sci USA 1999, 96:7336-7341

33. Petersen F, Bock L, Flad HD, Brandt E: A chondroitin sulfate proteoglycan on human neutrophils specifically binds platelet factor 4 and is involved in cell activation. J Immunol 1998, 161:4347-4355

34. Hirose J, Kawashima H, Yoshie O, Tashiro K, Miyasaka M: Versican interacts with chemokines and modulates cellular responses. J Biol Chem 2001, 276:5228-5234

35. Kawashima H, Hirose M, Hirose J, Nagakubo D, Plaas AH, Miyasaka $\mathrm{M}$ : Binding of a large chondroitin sulfate/dermatan sulfate proteoglycan, versican, to L-selectin, P-selectin, and CD44. J Biol Chem 2000, 275:35448-35456

36. Moore KL, Patel KD, Bruehl RE, Li F, Johnson DA, Lichenstein HS, Cummings RD, Bainton DF, McEver RP: P-selectin glycoprotein ligand-1 mediates rolling of human neutrophils on P-selectin. J Cell Biol 1995, 128:661-671

37. Zheng PS, Vais D, Lapierre D, Liang YY, Lee V, Yang BL, Yang BB: PG-M/versican binds to P-selectin glycoprotein ligand-1 and mediates leukocyte aggregation. J Cell Sci 2004, 117:5887-5895

38. Oka M, Edamatsu H, Kunisada M, Hu L, Takenaka N, Sakaguchi M, Kataoka T, Nishigori C: Phospholipase $\mathrm{C} \varepsilon$ has a crucial role in ultraviolet B-induced neutrophil-associated skin inflammation by regulating the expression of CXCL1/KC. Lab Invest 2011, 91:711-718

39. Mittal D, Saccheri F, Vénéreau E, Pusterla T, Bianchi ME, Rescigno M: TLR4-mediated skin carcinogenesis is dependent on immune and radioresistant cells, EMBO J 2010, 29:2242-2252

40. Smith JA: Neutrophils, host defense, and inflammation: a doubleedged sword. J Leukoc Biol 1994, 56:672-686 